\title{
ATM Retentate Detection Model of Texture Distinguish Optimization of HOG Operator
}

\author{
Zhen $\mathrm{Xu}^{1, \mathrm{a}}$, JianWei Lin ${ }^{1, \mathrm{~b}}$, Fan $\mathrm{Wu}^{1, \mathrm{c}}$, ZiBin $\mathrm{Xu}^{1, \mathrm{~d}}$ and Hanjie $\mathrm{Gu} \mathrm{u}^{1, \mathrm{e}}$ \\ ${ }^{1}$ Institute of Information Technology, Zhejiang Shuren University, Hangzhou 310014, China. \\ a61728419 @qq.com, b75799116 @qq.com, ${ }^{\mathrm{c}} 2846452$ @qq.com, ${ }^{\mathrm{d}} 1143701138$ \\ @qq.com, ${ }^{\mathrm{6}} 6035153 @ q q . c o m$
}

Keywords: retentate detection; HOG feature operator; Distinguish between grain; Background elimination; the characteristics of the block weighted.

\begin{abstract}
For the accuracy of traditional HOG feature detection operator in the application of ATM retentate detection is not high, this paper proposes a distinguish between optimization based on texture HOG feature detection operator ATM retentate detection model. First eliminate background of the original image by LBP operato, in order to highlight the local texture feature of detecting target, and then set a tolerance factor to eliminate the instability of LBP operator when neighborhood pixels change small, then the application of probability theory is adopted to optimize variance the similarity measures, and finally on the basis of the LBP background elimination, using the idea of entropy to HOG feature weighted in order to improve the detection accuracy. Experimental results show that the accuracy of proposed improved HOG feature detection based on texture distinguish optimization operator's is higher, the effect in the application of ATM machine retentate detection is better.
\end{abstract}

\section{Introduction}

In recent years, with the continuous development of our country finance informationization project and the advancement of the gold card project ATM occupies a pivotal position in the financial environment. Number of ATM machines is growing, and equipment placement position surrounding environment is more and more complex, so the difficulty of security monitoring, management is increasing [1]. At present, the ATM machine mainly relies on artificial to monitor, so that to screen event disputes, uncover the ATM crime cases [2]. However, this approach can only achieve later forensics, is likely to miss the best time to solve the event, not only time-consuming, even if the evidence can be found, damage may also be inevitable.

Many colleges and enterprises did research of ATM intelligent monitoring system, and obtained good results in some aspects. Liao [3] proposed the speed test the gaussian mixture model, but the large amount of calculation of the algorithm cannot satisfy the requirement of real-time. Caro [4] proposed boundary point method based on the determination of similarity matching, but the method for extracting target contour point of accurate and complete dependence is higher. Mao Xin [5] proposed the target mass analysis method based on human movement to realize the relic of detection. Seal just [6] proposed a detection algorithm based on time to judge the stranded object. Retentate detection algorithm uses background difference method to obtain the difference image of the current frame and background image, and does median filtering, binarization and morphological processing, to get the difference image binarization and the following processing. $\mathrm{Bi}$ [7] and others based on gaussian mixture distribution model, adopting different update rate cumulative mask update background model, established double background model. Based on the edge of the correlation matching algorithm, the identification and extraction prospect target, the spatial location of relic or move content is given and the police. $\mathrm{Li} \mathrm{[8]} \mathrm{and} \mathrm{others} \mathrm{proposed} \mathrm{a} \mathrm{retentate} \mathrm{or} \mathrm{move} \mathrm{content} \mathrm{detection}$ method on the basis of in average background updating model. Wang [9] and others studied surveillance and correlation analysis algorithm in the stranded in intelligent vide, when the monitoring objects in the scene the stranded, detect retentate and sends an alarm at the same time to extract the stranded key frames. Ye [10] and others put forward an effective algorithm for problems left over object detection in complex environment. Time to reach static threshold of briquette using 
gradient direction histogram (HOG) pedestrian detection, after rule out the possibility of reside pedestrians will mark it as a relic. And to detect the relic to accelerate splitting test features (FAST) local match, to overcome the pedestrian barrier, the influence of light on the results.

Based on traditional HOG feature detection operator in the defects existing in the practical application, this paper proposes a texture distinguish optimization HOG feature detection operator ATM retentate detection model, and its experimental simulation, verify the validity of the improvement strategy.

\section{Feature Detection Nodel based on HOG Operator}

The HOG feature detection operator [11] describes in the local area of a given image gradient direction and gradient intensity distribution and the change of situation, it can detect the target shape and outline a good show characteristics of the specific steps are as follows:

First, input the given image to the detection window, add image Gamma standardization and of color space standardization to preprocess the input image.

Then, calculate intensity and direction of the inside of the rectangular area of a given image gradient. $H$ expresses an image, $H(x, y)$ presents the gray value of a given image in pixels. Gradient calculation process is as follows:

$$
\begin{aligned}
& G_{x}(x, y)=H(x+1, y)-H(x-1, y) \\
& G_{y}(x, y)=H(x, y+1)-H(x, y-1)
\end{aligned}
$$

$G_{x}(x, y), G_{y}(x, y)$ represent a given image at $(x, y)$ pixel level in amplitude and the gradient in the direction of vertical gradient amplitude. The size of the gradient of the pixel points $(x, y)$ is:

$$
G(x, y)=\sqrt{G_{x}(x, y)^{2}+G_{y}(x, y)^{2}}
$$

Pixel gradient direction of the sample is:

$$
\theta(x, y)=\arctan \left(\frac{G_{y}(x, y)}{G_{x}(x, y)}\right)
$$

A given image window is divided into some of the same size of the cell, the pixel in each cell weighted voting to subordinate to the gradient direction histogram to get the gradient histogram space and direction. The L2 - norm to of normalizet he gradient histogram, its computation formula is as follows:

$$
v^{*}=\frac{v}{\sqrt{\|v\|^{2}+\varepsilon^{2}}}
$$

$v$ represents the normalized before feature vector, $\|v\|_{k}$ represents its $k$ order norm $k=1,2, \varepsilon$ represents a very small constant.

Finally, collect all the overlapping blocks in the detection window for HOG feature vector of a catch-all category.

HOG feature detection operator was applied to the ATM retentate tests, it reflects statistics of the retentate contour direction probability and in a certain area, easily affected by the texture noise, just relying on the retentate gradient information is easy to reduce the rates of detection of retentate, so in this paper, the defects were improved.

\section{Optimization of HOG Operator ATM Retentate Detection Model Based on Texture Distinguish}

\section{Texture Distinguish Optimization based on LBP Background Eliminating}

For HOG feature detection operator easily affected by the texture noise problem, this paper first USES the LBP operator [12] to eliminate background, in order to highlight the local texture feature of detecting target and enhance the robustness of the HOG operator. 
LBP mainly adopts $P$ pixel in the neighborhood of radius $R$ to describe the texture characteristics of pixels. LBP calculation such as type (6):

$$
\begin{gathered}
\operatorname{LBP}_{P, R}\left(x_{c}, y_{c}\right)=\sum_{p=0}^{P-1} s\left(g_{p}-g_{c}\right) \cdot 2^{p} \\
s(x)=\left\{\begin{array}{l}
1, x \geq 0 \\
0, x<0
\end{array}\right.
\end{gathered}
$$

$g_{c}$ represents pixel gray value of $\left(x_{c}, y_{c}\right), g_{p}$ represents each pixel gray in the circular field value with radius $R$.

However, when the neighborhood pixels change is small, LBP value calculation can get differences or fundamentally different results, so this paper sets a tolerance factor $a$ to eliminate the effect, the formula becomes:

$$
\operatorname{LBP}_{P, R}\left(x_{c}, y_{c}\right)=\sum_{p=0}^{P-1} s\left(g_{p}-g_{c}+a\right) \cdot 2^{p}
$$

Build the background model is $\left\{\vec{m}_{0}, \vec{m}_{1}, \ldots, \vec{m}_{N-1}\right\} . \vec{m}_{i}$ represents $i$ LBP texture histogram in background model, it uses the current pixel as the center, $R$ radius to calculate, each histogram has a weight $w_{i}$.

Background deduction process is: compare the current pixel histogram $\vec{h}$ and the model of the $n$ background of the histogram individually, if a variable of a histogram and the similar degree is less than the setting threshold, then the pixels was sentenced to prospect, otherwise sentenced as the background.

Among them, the way of determine $n$ as shown in type (9) :

$$
n=\underset{b}{\arg \min }\left(\sum_{b=0}^{N-1} w_{b}>T_{n}\right)
$$

Histogram $\vec{a}, \vec{b}$ similarity calculation is type (10):

$$
\cap(\vec{a}, \vec{b})=\min \sum_{n=0}^{N-1}\left(a_{n}, b_{n}\right)
$$

Then, the application of probability theory is adopted to similar measurement accuracy of the optimization. According to the application of probability theory, the variance is defined as: if $\xi$ is a random variable, if $E[\xi-E(\xi)]^{2}$ exist, it is variance of $\xi$ as $D(\xi)$.

$$
D(\xi)=E[\xi-E(\xi)]^{2}
$$

Among them $E(\xi)$ is the mathematical expectation of a random variable:

$$
E(\xi)=\sum_{i=1}^{\infty} x_{i} p_{i}
$$

The above formula is a general definition of variance of a random variable, in ATM retentate recognition, for each image sequence, variances are defined as follows:

$$
\operatorname{var}^{k}=\frac{1}{N} \cdot\left[\sum_{i=1}^{N} d\left(S_{i}^{k}, S^{k}\right)\right]^{2}
$$

Among them, $\operatorname{var}^{k}$ represents the $k$ child of variance of image sequence after dividing, $N$ represents total number of training samples, $s$ represents sub image the average of all training samples:

$$
S=\frac{1}{N} \cdot \sum_{i=1}^{N} S_{i}
$$

$S^{k}$ represents the $k$ sub image of all the sample average image $S, S_{i}^{k}$ represents the $k$ sub image of the $i$ sample, $d\left(S_{i}^{k}, S^{k}\right)$ represents all the sample average image $S$ and the similarity measure distance between samples $S_{i}$ corresponding to the sub image, $k=1,2, \ldots, n^{2}$. 
So, as long as the selected a similar measure as distance $d\left(S_{i}^{k}, S^{k}\right)$, for any image classification, can be in accordance with the above formula to calculate the corresponding to the variance of child image sequence, this variance as each child image of the local weights of texture feature, and then according to the corresponding weight on the similarity level fusion, finally, to this kind of background measure to eliminate.

\section{The HOG Feature Weighting Based on the Entropy}

On the basis of removal by LBP background, in order to further improve the precision of the HOG feature detection algorithm, this paper adopts the idea of entropy weighted on the HOG feature block.

First calculate the entropy of the characteristics of each block, and then multiply the calculated entropy as a right value into corresponding characteristic block HOG feature vector, the normalization processing. Each of these characteristics of block of entropy calculation is as follows:

$$
\begin{gathered}
E=-\sum_{i=1}^{c \times l} P_{i} \log _{2} P_{i} \\
P_{i}=\frac{h_{i}}{\sum_{t=1}^{c \times l} h_{t}}, i=1,2, \ldots, c \times l
\end{gathered}
$$

$h_{i}$ and $P_{i}$ represent $i$ gradient histogram amplitude and the amplitude percentage of the total amplitude of the characteristics block, $c$ and $l$ represent the cell number of characteristics the block and number of the gradient histogram feature of each cell.

Among them, if $P_{i}=0, \log _{2} P_{i}$ is negative infinitesimal, so we provisions when $P_{i}=0$ :

$$
P_{i} \log _{2} P_{i}=0
$$

By the above method, add the entropy into the characteristics of the block of HOG features, can effectively improve the accuracy of the original algorithm..

\section{Algorithm Performance Simulation}

In order to verify the effectiveness of the improved algorithm proposed in this paper, do simulation experiments. Experimental environment is: the CPU for the Intel (R) Core (TM) i5-3470, faster 3.2 G, memory 8 G, using Visual Studio 2008 development environment, and computer vision library Open CV 2.0.

This experiment takes hat, mobile phones, stickers, for example, for a period of ATM real-time monitoring video retentate tests, which is shown in figure 1 that when hat is retentate the traditional HOG operator and improve the algorithm of test results, figure 2 that when phone is the retentate the test results of two kinds of algorithm, figure 3 that when stickers is retentate test the results of both methods (left for the traditional algorithm, the improved algorithm has the graph).
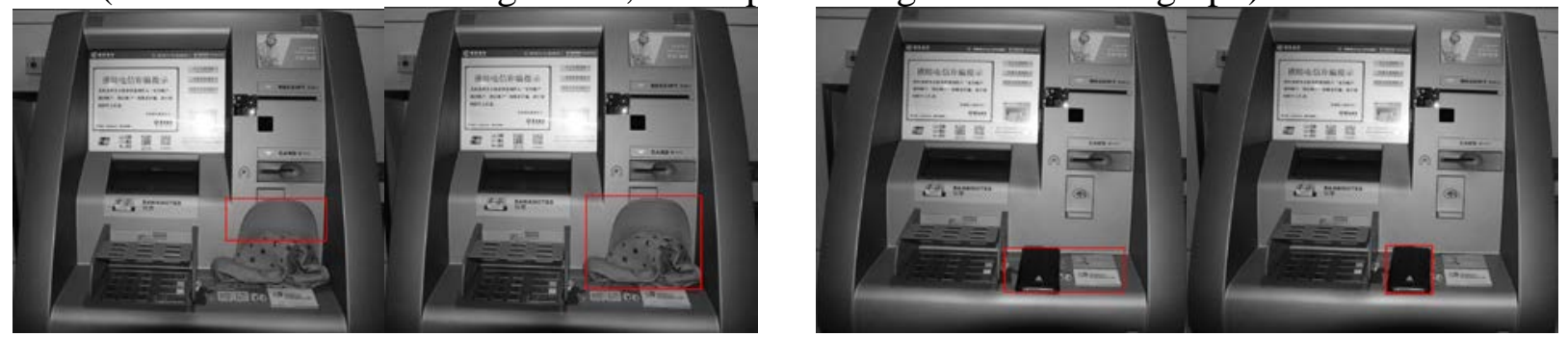

Fig1 Existence detection result was when the hat Fig2 Existence detection result thereof to the phone when 


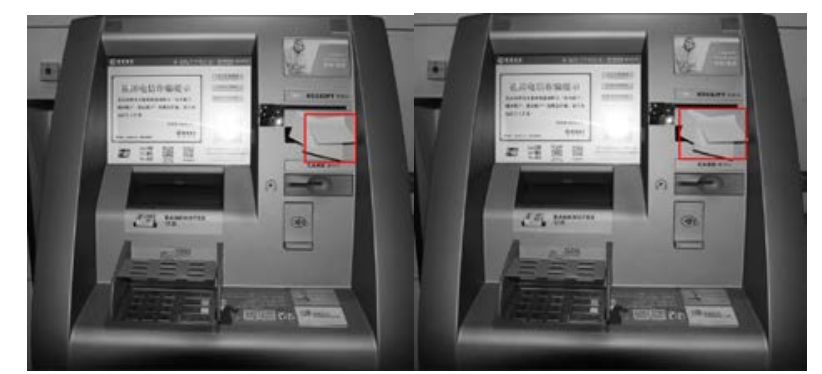

Fig3 The test results for the stickers was stranded when

From the simulation experiments, this paper proposes the improved HOG feature detection based on texture distinguish optimization operator's accuracy is higher, in the application of ATM retentate detection effect is better.

\section{Summary}

With the development of the society, the retentate as ATM security problems get more and more attention, the original monitoring system has been unable to meet the needs of people.Based on traditional HOG feature detection operator in the defects existing in the practical application, this paper proposes a texture distinguish optimization based on ATM retentate HOG feature detection operator testing model, the experimental simulation results show that the proposed improved algorithm in ATM retentate detection accuracy is higher.

\section{Acknowledgement}

This work was supported by the Education of Zhejiang Province (Grant No. Y201225667), and the Department of Science and Technology of Zhejiang Province (Grant No.2014C31065), and the Department of Science and Technology of Zhejiang Province (Grant No. 2015C31091).

\section{References}

[1] Y.P Tang, He L Zu. Application of Dynamic Image Understanding in ATM Intelligent Surveillance. Computer Measurement \& Control,2013,(6):1110-1112.

[2] Y.P Tang, J Ye, LIANG Yigong. ATM intelligent surveillance based on ODVS. Journal of Zhejiang University of Technology,2012,(1):26-32.

[3] H Liao, J Chang, L Chen. A Localized Approach to Abandoned Luggage Detection with Foreground-Mask Sampling[J].5th IEEE Int. Conference on AVSS, Santa Fe, New Mexico, USA: IEEE Press,2008:132-139.

[4] C.L Caro, J.C SanMiquel, J.M Martine. Discrimination of Abandoned and Stolen Object based on Active Contours. IEEE International Conference on Advanced Video and Signal-based Surveillance (AVSS),2011,(3):101-106.

[5] X Mao, P.J Chen, G Tian, QI Feihu. Component-based Pedestrian Detection with Monocular Moving Camera. Journal of System Simulation.2012,18(10):2906-2910.

[6] Z.Y Huang, L.X Li, J.H Wu. A Face Detection Technology Based On HSV Color Segmentation And Fuzzy Cascade Classifier Algorithm. Computer Applications and Software.2014,31(4):197-200.

[7] G.L Bi, J Zhao, Z.J Xu. An Detection Algorithm for Abandoned and Moved Objects Used in Intelligent Surveillance Video[J].Microelectronics \& Computer.2013,30(12):27-30.

[8] S.J Li, B.M P, B.Y He, W.W Wang. An Abandoned/Removed Object Detection Method Based on Image Block. Mini-micro Systems.2014,35(1):142-147. 
[9] P Wang, X.C Zhu. Association Analysis Algorithm Between Abandoned Object and Human in Intelligent Video Surveillance. Tv Engineering,2013,35(3):101-105.

[10] L.R Ye, S.H He, L.C Zhao. An abandoned object detection algorithm in complex environments. Computer Engineering \& Science.2015,37(5):986-992.

[11] W.X Li, F Pan, X.M Ren. Multi-Definition Template of HOG for Pedestrian Detection.Computer Simulation.2015,32(7):398-401.

[12] X.F Liu. Face Recognition Method Based on Neighborhood Correlation Modified LBP Operator. Journal of Jilin University: Sci Ed.2015,53(4):687-692. 